\title{
Políticas públicas y empoderamiento de mujeres indígenas en Ensenada, Baja California
}

\author{
Diana G. Robinson-Trápaga \\ Universidad Autónoma de Baja California, México \\ Isis A. Díaz-Carrión \\ Universidad Autónoma de Baja California, México \\ Sergio Cruz-Hernández \\ Universidad Autónoma de Baja California, México
}

Recibido: 22 de junio de 2019

Aprobado: 4 de octubre de 2019

\section{Resumen}

Las mujeres indígenas experimentan una triple discriminación a nivel mundial por razón de género, origen étnico y situación de pobreza. Para atender esta problemática, los gobiernos diseñan programas de políticas públicas con perspectiva de género que buscan integrarlas al trabajo productivo e impulsarlas hacia un proceso de empoderamiento en los espacios público y privado de su vida.

En México, durante la presidencia de Enrique Peña Nieto, la Comisión Nacional para el Desarrollo de los Pueblos Indígenas (CDI) a través del Programa para el Mejoramiento de la Producción y Productividad Indígena (PROIN) otorgaba apoyos a grupos productivos de mujeres indigenas con el objetivo de aumentar sus ingresos monetarios y no monetarios, y mejorar sus condiciones de vida.

El propósito de esta investigación es medir el empoderamiento en los grupos de mujeres indígenas beneficiarias en el municipio de Ensenada, Baja California, de 2014 a 2017. Se aplica una metodología cuantitativa. 
Se destaca el importante papel de la participación de las mujeres en programas para potenciar el empoderamiento. No obstante este significativo avance, se establece como prioritario ofrecer acompañamiento y capacitación con perspectiva de género, dado el sistema patriarcal sociocultural que prevalece y constriñe su proceso de empoderamiento.

Palabras clave: empoderamiento, microempresa social indígena, mujer indígena.

Clasificación JEL: J15, J16. 


\begin{abstract}
Indigenous women experience discrimination worldwide due to gender inequity, ethnic origin and poverty. Gender public policies are designed to mitigate such situation, drive women into an empowerment process in public and private spheres and integrate them into productive work.
\end{abstract}

In Mexico, during Enrique Peña Nieto's presidency, the Comisión Nacional para el Desarrollo de los Pueblos Indígenas, CDI (National Commission for the Development of Indigenous People) through the Programa para el Mejoramiento de la Producción y Productividad Indigena, PROIN (Indigenous Production and Productivity Improvement Program), granted support to productive groups of indigenous men and women, to increase their monetary and / or non-monetary income, empower women and girls, and improve their life conditions.

The purpose of this research is to measure empowerment in indigenous women's productive groups benefited by PROIN from 2014 to 2017, in the Ensenada Municipality, Baja California, Mexico. A Quantitative method is applied.

Among our main findings, we highlight the importance of participating in these productive groups to promote women empowerment; however, women need gender perspective accompaniment and guidance to deal with the sociocultural patriarchal system, which constrains their empowerment.

Keywords: empowerment, indigenous social microenterprise, indigenous woman.

JEL Classification: J15, J16. 


\section{Introducción}

Las mujeres indígenas sufren una triple discriminación por su origen étnico, situación de pobreza e inequidad de género (García-Canclini, 2012; ONU, 2007; Serrano-Barquín, et al., 2013; Zarza-Delgado y SerranoBarquín, 2013); la falta de educación, el poco acceso a oportunidades y el sistema patriarcal ancestral sociocultural abonan a esta situación.

Estas problemáticas se abordan a nivel internacional desde la Organización de las Naciones Unidas, en los 17 Objetivos de Desarrollo Sostenible de la Agenda 2030 (ONU, 2015), específicamente en el objetivo «1. Fin de la pobreza» y objetivo «5. Igualdad de género». En México, el gobierno federal se suma a estos esfuerzos a través de programas de política pública con perspectiva de género que buscan integrar e impulsar a las mujeres y conducir al mejoramiento de sus condiciones de vida, empoderamiento, bienestar social, empleo y mejora económica (Gobierno de la República, 2014; Gobierno de la República, 2013).

La Comisión Nacional para el Desarrollo de los Pueblos Indígenas (CDI) era, hasta el 2018, durante la gestión del presidente Enrique Peña Nieto, la instancia que se encargaba de poner en marcha los programas que apoyan e impulsan el trabajo productivo de la población indígena mediante subsidios o microcréditos. El Programa para el Mejoramiento de la Producción y la Productividad Indígena (PROIN) se dirige a grupos de trabajo, sociedades o empresas indígenas que participan en proyectos productivos. La Modalidad 5.1.1 de dicho programa se dirige en específico a mujeres indígenas (Gobierno de la República, Comisión Nacional para el Desarrollo de los Pueblos Indígenas, 2017).

Los resultados del impacto que generan estos programas son heterogéneos. La CONEVAL (2015) realiza una evaluación desde su diseño, implementación y operatividad, sin embargo, hacen falta valoraciones del impacto económico y sociocultural en la vida de las beneficiarias (Gobierno de la República, 2014).

El objetivo de este trabajo es analizar el proceso de empoderamiento de las mujeres indígenas beneficiarias en diferentes dimensiones de su vida, a partir de la participación en los grupos de trabajo. Se consideran los grupos beneficiados por el PROIN de 2014 a 2017 en el municipio de Ensenada, Baja California.

La valoración de los resultados del programa en cuanto al proceso de empoderamiento de las mujeres indígenas beneficiarias en diferentes dimensiones permite corroborar si ha tenido un impacto positivo en su vida y ello resuelve la necesidad que apunta el Plan Especial de los Pueblos Indígenas (Gobierno Federal, 2014) de realizar una investigación que valore el 
programa desde el cambio sistémico sociocultural con una perspectiva de género y no solo de su diseño, gestión e implementación.

Se aplica una metodología cuantitativa, se determinan las variables de estudio a considerar y se construyen índices para el cálculo de empoderamiento en cada una de las dimensiones.

\section{Marco teórico}

\section{Concepto de empoderamiento}

El concepto de empoderamiento aparece en la agenda política del feminismo en la década de 1970. El pensamiento feminista surge como un cuestionamiento político al discurso androcéntrico y de dominación sobre la mujer, que las caracterizaba como receptoras pasivas encargadas del hogar (Ramírez-Salgado, 2017), mientras que los hombres aparecían como proveedores con un trabajo asalariado.

Por ello, desde finales del siglo pasado, las mujeres buscan el desarrollo y atención a sus necesidades e inclusión en los procesos económicos (Aguinaga, et al., 2011; Gil, 2017; Pont, 2010; Virgilí, 2014). La teoría feminista contribuye de manera extensa al estudio de la participación económica de las mujeres (Ramírez-Salgado, 2017), hace notar los mecanismos de discriminación en el mercado laboral (Rodríguez, 2015), a la vez que promueve relaciones de poder más equilibradas.

Para comprender el concepto, primero definimos lo que se entiende por poder, para lo cual se retoma la tipología de Lukes (2005), según Mayoux (2000) y Rowlands (1997), y después se define el concepto de empoderamiento de acuerdo con diversas autoras y algunas aproximaciones para su valoración.

Mayoux (2000) define empoderamiento como un proceso multidimensional vinculado con las cuatro relaciones de poder de Lukes (2005): poder desde dentro, poder sobre, poder para y poder con. «Poder desde dentro» se refiere a la conciencia de las mujeres de articular sus propias estrategias y aspiraciones. «Poder para» permite a las mujeres desarrollar las habilidades necesarias y acceder a los recursos para alcanzar sus objetivos. «Poder con» se refiere a organizar sus intereses colectivos, organizarlos para alcanzarlos y vincularse con organizaciones de otras mujeres y hombres hacia el cambio. «Poder sobre» alude al cambio en poder y recursos que constriñen sus aspiraciones y su habilidad para alcanzarlos.

Estas relaciones de poder operan en diferentes esferas de la vida: económica, social, política, y en diferentes niveles: individual, en el hogar, en la comunidad, el mercado y en las instituciones. Por su parte, Lagarde (2005) 
define el poder como la capacidad de tomar decisiones sobre la vida del otro(a). Quien ejerce el poder obliga, prohíbe, castiga, enjuicia y perdona.

Rowlands (1997) explica el concepto del empoderamiento de las mujeres desde tres esferas: la personal, la de relaciones cercanas y la colectiva. La primera se refiere a la autoestima, confianza y seguridad en sí mismas, a la habilidad de participar, organizarse, expresarse, idear. La esfera de las relaciones cercanas es la capacidad de transformar las relaciones para poder influir, negociar y tomar decisiones que beneficien al grupo; cuando logran cambios en esta dimensión, es probable que se impacte en otros aspectos de su vida. La colectiva se caracteriza por el trabajo colaborativo e incluye la participación política.

Young (1997) identifica dos términos: condición y posición de las mujeres. Condición es el estado material en el que se encuentra, su pobreza, la excesiva carga de trabajo, educación, falta de acceso a la tecnología. Posición se refiere a su ubicación social y económica con respecto a los hombres. Rahman (2013), por su parte, propone el empoderamiento como un concepto neutral, que implica transformación de las relaciones de género, de un plano jerárquico a uno igualitario y no desde la posición de poder de las mujeres; empoderamiento de género como categoría incluyente, que motiva un equilibrio entre las relaciones de género.

Para Kabeer (1999) el empoderamiento de las mujeres es la habilidad de tomar decisiones de vida estratégicas en ámbitos en que anteriormente le han sido negadas; se relaciona con la toma de decisiones en el espacio público y privado, en las dimensiones personal, familiar, económica, política, legal y sociocultural.

El empoderamiento de las mujeres es un proceso a través del cual estas empiezan a ser conscientes de su propio poder, de su capacidad de tomar decisiones sobre su propia vida (Díaz-Carrión, 2012; Kabeer, 2001; Longwe y Clarke, 1994; Mayoux, 2000; Malhotra, Schuler y Boender, 2002; Rowlands, 1997; Tuñón-Pablos, 2010), del lugar que ocupan en el hogar y en la sociedad, y se manifiesta en el ámbito privado y público.

A nivel personal, incide en la autoestima, la familia, las dinámicas dentro del hogar, es decir, en cómo son las relaciones de poder con la pareja, hijos, hijas, familia política y la carga del trabajo doméstico que realizan (Mayoux, 2000; Kabeer, 2001; Malhotra, Schuler y Boender, 2002; Rowlands, 1997). Stromquist (1997) plantea el empoderamiento como un proceso mediante el cual cambia la distribución del poder, incluye componentes cognitivos, psicológicos, políticos y económicos.

Tradicionalmente, las mujeres se encargan de la casa y los hijos, algo aprendido por herencia y constructos sociales preestablecidos, dejándose a sí mismas en último lugar, al servicio de las demás personas (Alberti-Manzanares, 
et al., 2014). Al entrar en un proceso de empoderamiento, empiezan a darse cuenta de sus opciones y capacidad de tomar decisiones, valoran su tiempo y reconocen su autonomía; es algo que inicia desde la propia consciencia, a un nivel interior, por lo que es necesario que el ambiente lo propicie por medio de la información, que conozcan sus derechos y haya programas económicos y sociales que las impulse (Mathur y Agarwal, 2016; AguilarPinto, et al., 2017).

Hace ya varias décadas que se analizan programas de política pública y estrategias que buscan impulsar a las mujeres rurales hacia un proceso de empoderamiento en la esfera pública y privada (Banerjee y Hackson, 2017; Martínez-Corona, 2000; Díaz-Cervantes, 2015; Rahman, 2013). Los resultados difieren según los programas, el contexto sociocultural, la situación personal, familiar y económica de cada una de las mujeres. El proceso se ha valorado en diferentes países a través de propuestas de modelos que integran diversas dimensiones de la vida de las mujeres.

Los grupos productivos de mujeres indígenas apoyados por el PROIN son emprendimientos que se forman a partir de la subvención del gobierno con el propósito de conducir a las mujeres a su propio proceso de empoderamiento, así como mejorar su vida y la de sus familias al brindar un recurso económico y acompañamiento en la gestión del grupo.

Investigaciones sobre grupos productivos apoyados por microcréditos o subsidios del gobierno en México sustentan el hecho de que, cuando las mujeres rurales e indígenas se agrupan y emprenden un proyecto para realizar un trabajo productivo, se observan dinámicas de empoderamiento dentro de la organización y se produce un impacto económico y social (Banerjee y Jackson, 2017; García-Horta y Zapata-Martelo, 2012; Meza, et al., 2002; Rivas-Ángeles, et al., 2015; Tuñón-Pablos, 2010; Zapata-Martelo y MercadoGonzález, 1996). Las mujeres empiezan a tomar decisiones sobre cómo invertir el dinero, en qué gastar, qué comprar, e inician una construcción de redes entre ellas mismas y con el exterior, al relacionarse comercialmente con proveedores y vendedores (Zapata, et al., 2008). El trabajo productivo las lleva a generar un ingreso adicional para ellas y para sus familias, gracias a lo cual empiezan a intervenir en la toma de decisiones sobre el gasto del hogar y a transformar sus relaciones familiares. Las mujeres asignan mayoritariamente el dinero a mejorar la vida de sus hijos, administran el dinero para el bienestar de la familia, se ocupan de la educación, la salud y de la vivienda (Yunus, 2007).

Zapata-Martelo y Mercado-González (1996) presentan que los grupos productivos de mujeres que tienden a convertirse en microempresas deben caracterizarse como microempresas sociales debido a que, además de buscar rentabilidad económica y beneficio social, crean espacios para generar empoderamiento. 
La organización productiva les permite salir del espacio doméstico. Los logros se ubican a nivel personal, comunitario, familiar y organizacional.

Por su parte, Lemaître y Helmsing (2012) analizan las actividades económicas de 15 microempresas sociales en las favelas en Río de Janeiro, Brasil; estudian las organizaciones desde tres perspectivas: los logros, las relaciones económicas y las relaciones internas. Los logros deben darse en la dimensión económica, social, política y medioambiental. En cuanto a las relaciones económicas, se consideran tanto las monetarias (ingreso) como las no monetarias, que no representan un ingreso económico pero tienen un valor para la organización, como el voluntariado, la reciprocidad, el trabajo doméstico. Las relaciones internas se refieren a las estructuras de poder que se establecen en la microempresa.

La actividad económica no es central; lo es la importancia de iniciar a los participantes en un proceso de empoderamiento; destaca la construcción de redes y solidaridad entre los participantes. El ingreso que perciben es bajo y el valor social que se produce es alto (Lemaître y Helmsing, 2012, ZapataMartelo y Mercado-González, 1996).

\section{Metodología}

Para la medición del empoderamiento, se aplica una metodología cuantitativa a través de índices en cuatro dimensiones. La hipótesis de la investigación es la siguiente:

$H$ : Entre las mujeres indígenas que integran los grupos productivos del PROIN se generan procesos de empoderamiento en las dimensiones económica, personal, familiar y sociocultural, a partir de su participación en los grupos de trabajo.

Se retoman dimensiones propuestas por diversos autores que afirman que el empoderamiento es un proceso multidimensional (Malhotra, Schuler y Boender 2002; Foley, 1997; Gigler, 2011; Haughy Talwar, 2014; Malhotra, Schuler y Mayoux, 2000; Stromquist, 1997).

Se diseña el instrumento para medir el empoderamiento de mujeres indígenas beneficiarias del Programa para el Mejoramiento de la Producción y Productividad Indígena (PROIN) para la recolección de datos. La primera sección del instrumento incluye preguntas de datos sociodemográficos, como edad, estado civil, número de hijos, ingresos por el grupo de trabajo apoyado por el PROIN y otros ingresos. La segunda se compone de reactivos que miden cada una de las dimensiones de estudio. Las respuestas se valoran a partir de cinco niveles en escala de Likert, donde 1 corresponde a totalmente en desacuerdo; 2 a en desacuerdo; 3 a ni de acuerdo ni en desacuerdo; 4 de acuerdo, y 5 totalmente de acuerdo. 
Los datos se utilizan para la obtención de índices de empoderamiento por dimensión y un índice total de empoderamiento por mujer beneficiaria. A partir de estos índices, se calcula el nivel de empoderamiento total por grupo y por dimensión en cada mujer.

El cuestionario se aplica de manera personal a las mujeres beneficiarias que participan activamente en los grupos productivos apoyados por el PROIN de 2014 a 2017, en el municipio de Ensenada, Baja California, México. Se solicitan expedientes al Instituto Nacional de Transparencia, Acceso a la Información y Protección de Datos Personales (INAI) y estos son proporcionados por la Unidad de Transparencia de la Comisión Nacional para el Desarrollo de los Pueblos Indígenas el 14 de febrero de 2018, Folio UT/2018/OF/0053.

\section{Delimitación de la investigación}

Según expedientes del INAI, se registran 23 grupos productivos, 130 mujeres indígenas beneficiarias; sin embargo, al momento de realizar la investigación de campo (julio-agosto 2018), solo permanecen 40 mujeres trabajando activamente en los grupos productivos. Se registra una alta deserción, el $22 \%$ de los grupos que empezaron en 2014 continúa en operación; $71 \%$ de los de 2015; $75 \%$ de 2016 y $100 \%$ de 2017 .

Al empezar el programa en 2014, nueve grupos son beneficiados; posteriormente se subvenciona a seis grupos cada año. Al comparar las cantidades otorgadas de 2014 a 2017, se observa que el monto total asignado disminuye en $60 \%$ a lo largo de los cuatro años. De 2014 a 2015 se reduce en $40 \%$; 2016 se mantiene y en 2017 se observa una disminución adicional de $20 \%$. El presupuesto de la CDI depende del monto asignado para los programas de subsidio del Ramo Administrativo 47, exclusivos para el desarrollo de los pueblos indígenas, y es presupuesto del gobierno federal (Gobierno de la República, 2017).

De los 23 grupos beneficiados, trece se encuentran en operación y, de estos últimos, tres permanecen con el número de integrantes totales registradas en expedientes. Los demás grupos aún activos operan con menos mujeres que las que firman en el acta de constitución del grupo de trabajo; se observa que 90 mujeres desertaron de los proyectos, esto es $69 \%$ del total de las beneficiarias, y permanece $31 \%$, es decir, 40 integrantes trabajan activamente al momento de la investigación de campo $(n=40)$. Se aplica la encuesta de manera personal a la totalidad de las mujeres.

La actividad económica a la que se dedican, de acuerdo con el Sistema de Clasificación Industrial de América del Norte, SCIAN (2018) son: sector 11, agrícola, pecuaria, forestal, pesquera, acuícola; sector 43-46, comercio al por menor y al por mayor; sectores 72 y 81 , servicios; y sectores $30-31$, industria manufacturera. 


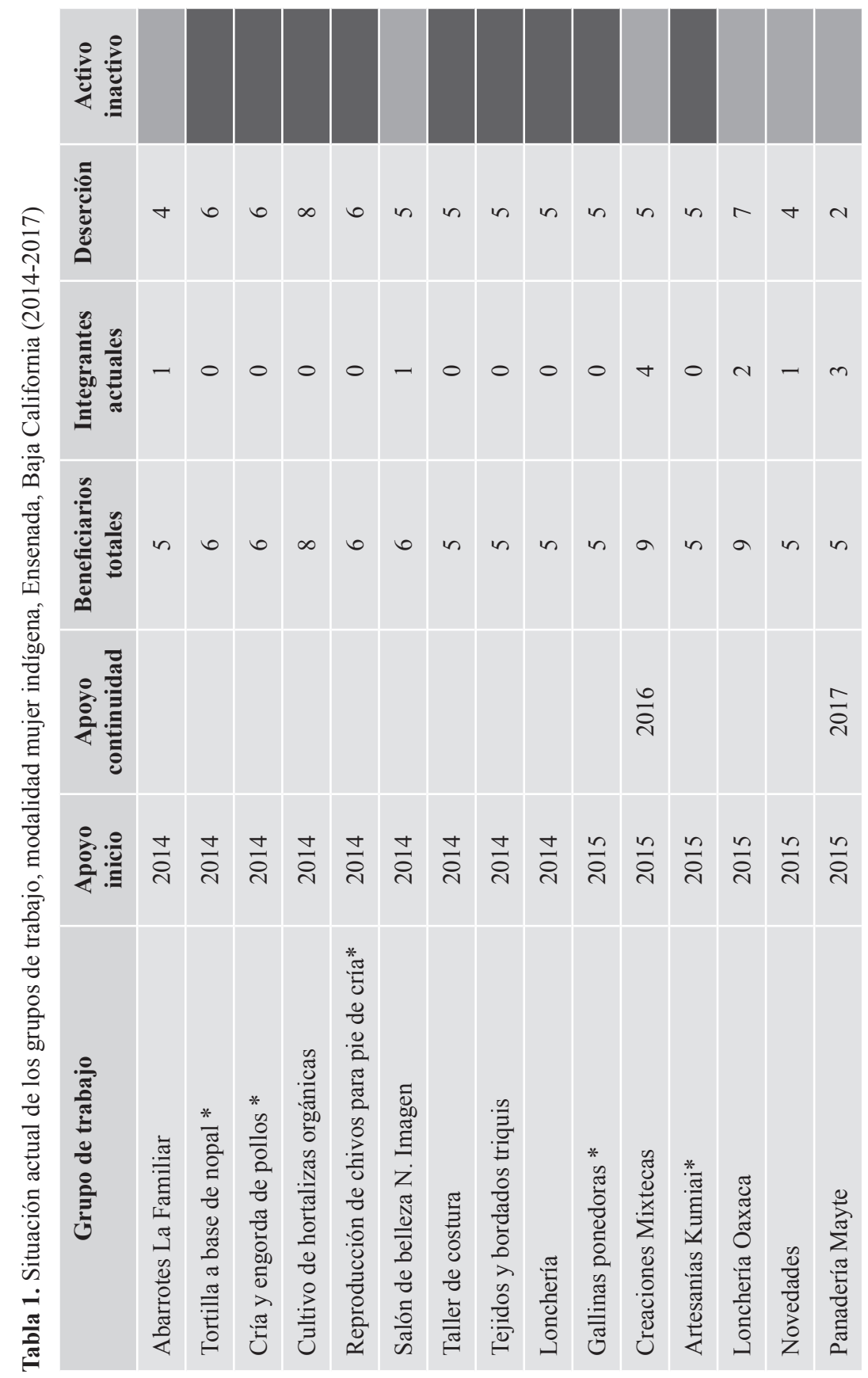




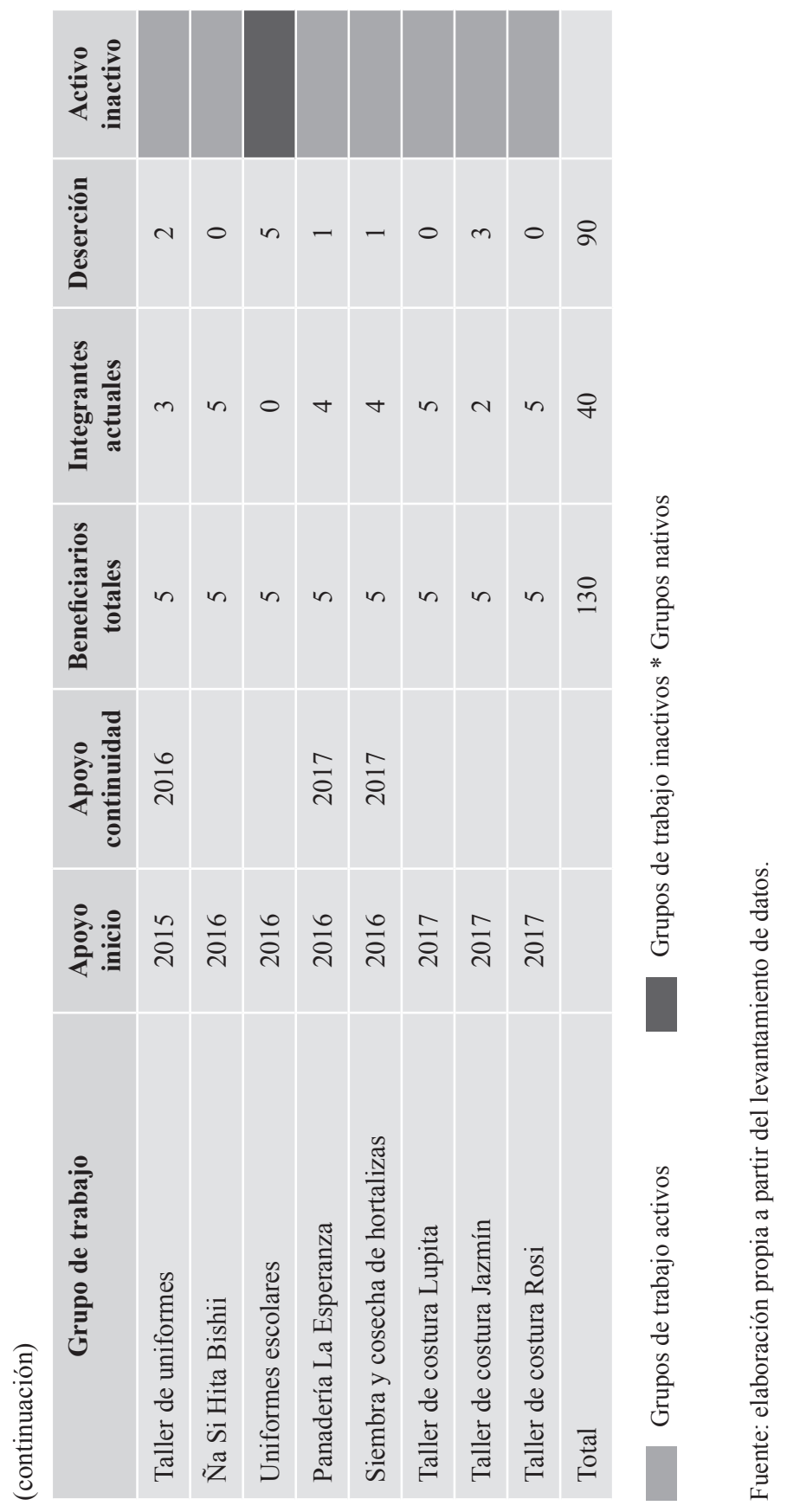


La tabla 1 presenta el total de los grupos beneficiarios, los grupos que han desertado del programa y los que persisten. Se detalla el número de mujeres que desertan, las integrantes actuales y los años en que reciben el apoyo. El grupo Creaciones Mixtecas es el único que ha recibido tres apoyos: el de inicio, continuidad y consolidación; uno con POPMI (Programa de Organización Productiva para Mujeres Indígenas) en 2013, programa que antecede a PROIN, y dos apoyos de PROIN. Ahora este grupo es elegible para solicitar apoyo al financiamiento. Se registra que los cinco grupos nativos beneficiados se desintegraron (ver tabla 1).

\section{Dimensiones del estudio}

En este apartado se detallan las variables que integran cada una de las dimensiones de estudio.

\section{Dimensión económica}

La dimensión económica determina la importancia que le asignan las mujeres específicamente al ingreso y nos permitirá dirimir qué tan empoderadas se encuentran en este campo. Esta dimensión se integra por tres variables: acceso y control de recursos (ACR); ingreso (I) y satisfacción de necesidades básicas (SN). Se construye a partir de once reactivos que nos permiten conocer la percepción de las mujeres en esta dimensión (ver tabla 2).

Tabla 2. Variables de estudio de la dimensión económica

\begin{tabular}{|c|c|}
\hline \multicolumn{2}{|c|}{ Dimensión económica } \\
\hline Variable & Descripción \\
\hline $\begin{array}{l}\text { Acceso y control de } \\
\text { recursos }\end{array}$ & $\begin{array}{l}\text { Decisión sobre uso del dinero. } \\
\text { Mayores ingresos propios, más satisfactores. } \\
\text { Opinión compartida sobre gastos. } \\
\text { Seguridad por obtención de ingresos. }\end{array}$ \\
\hline Ingreso & $\begin{array}{l}\text { Remuneración económica constante. } \\
\text { Remuneración suficiente. } \\
\text { Autonomía para cubrir los gastos del hogar. } \\
\text { Administración del ingreso. } \\
\text { Capacidad de ahorro a partir del trabajo en el grupo. }\end{array}$ \\
\hline $\begin{array}{l}\text { Satisfacer } \\
\text { necesidades básicas }\end{array}$ & $\begin{array}{l}\text { Vivir mejor a partir del trabajo. } \\
\text { Bienestar económico para la familia. }\end{array}$ \\
\hline
\end{tabular}

Fuente: elaboración propia a partir de las dimensiones propuestas por diversos autores: Alkire, et al. (2012), Foley (1997), Gigler (2011), Haugh y Talwar (2014), Lemaître y Helmsing (2012), Malhotra, Schuler y Boender (2002), Zapata-Martelo y Mercado-González (1996). 
Se refiere al desarrollo de las mujeres en lo individual, a nivel personal. Se construye a partir de once reactivos que consideran la toma de decisiones personales (TDP); la movilidad (M), que implica libertad para desplazarse de un lugar a otro libremente; la seguridad por el trabajo productivo (ST) y la autoconfianza (AC); (ver tabla 3).

Tabla 3. Variables de estudio de la dimensión personal

\begin{tabular}{|l|l|}
\hline Dimensión personal & Descripción \\
\hline Variable & $\begin{array}{l}\text { Seguridad al tomar decisiones personales. } \\
\text { Toma de decisiones } \\
\text { personales }\end{array}$ \\
\hline $\begin{array}{l}\text { Autonomía en la toma de decisiones. } \\
\text { Determinación para decidir. }\end{array}$ \\
\hline Movilidad & $\begin{array}{l}\text { Libertad para ir de un lugar a otro. } \\
\text { Autonomía para decidir cuándo y a dónde salir. }\end{array}$ \\
\hline $\begin{array}{l}\text { Seguridad por el } \\
\text { trabajo }\end{array}$ & $\begin{array}{l}\text { Confianza por desempeñar un trabajo. } \\
\text { Valoración del trabajo por el grupo doméstico. }\end{array}$ \\
\hline Autoconfianza & $\begin{array}{l}\text { Seguridad para expresarse libremente. } \\
\text { Confianza en sí misma. } \\
\text { Capacidad y seguridad en sus metas. }\end{array}$ \\
\hline
\end{tabular}

Fuente: elaboración propia a partir de las dimensiones propuestas por diversos autores: Alkire, et al. (2012), Foley, (1997), Gigler (2011), Haugh y Talwar (2014), Lemaître y Helmsing (2012), Malhotra, Schuler, Boender (2002), Zapata-Martelo y Mercado-González (1996).

\section{Dimensión familiar}

Se refiere a la situación de las mujeres dentro del núcleo familiar, su relación con sus hijas(os), pareja, familia política. Se construye a partir de las variables equidad (E), toma de decisiones dentro de la familia (TDF) y trabajo doméstico (TD). Se obtiene a partir de nueve reactivos (ver tabla 4). 
Tabla 4. Variables de estudio de la dimensión familiar

\begin{tabular}{|c|c|}
\hline \multicolumn{2}{|l|}{ Dimensión familiar } \\
\hline Variable & Descripción \\
\hline Equidad & $\begin{array}{l}\text { Apoyo de la pareja. } \\
\text { Misma educación para hijos e hijas. } \\
\text { Igualdad entre hombres y mujeres de la casa. } \\
\text { Autovaloración del trabajo doméstico y productivo. } \\
\text { Aceptación y apoyo de la familia con respecto al trabajo } \\
\text { de las mujeres. }\end{array}$ \\
\hline $\begin{array}{l}\text { Toma de decisiones } \\
\text { familiares }\end{array}$ & $\begin{array}{l}\text { Decisiones en pareja en cuanto a gastos del hogar. } \\
\text { Decisiones en pareja sobre la familia. }\end{array}$ \\
\hline Trabajo doméstico & $\begin{array}{l}\text { Trabajo del hogar repartido en la familia. } \\
\text { Niños y niñas, labores indistintas en el trabajo del hogar. }\end{array}$ \\
\hline
\end{tabular}

Fuente: elaboración propia a partir de las dimensiones propuestas por diversos autores: Alkire, et al. (2012), Foley, (1997), Gigler (2011), Haugh y Talwar (2014), Lemaître y Helmsing (2012), Malhotra, Schuler, Boender (2002), Zapata-Martelo y MercadoGonzález (1996).

\section{Dimensión sociocultural}

Mide los cambios que perciben las mujeres en su comunidad en cuanto a la toma de decisiones en el grupo (TDG), equidad en la comunidad (EC), movilidad por el trabajo (M) y construcción de redes (R), es decir, relaciones que se entablan con otras mujeres, proveedores, vendedores. Se determina con base en 12 reactivos (ver tabla 5).

Tabla 5. Variables de estudio de la dimensión sociocultural

\begin{tabular}{|l|l|}
\hline \multicolumn{2}{|l|}{ Dimensión sociocultural } \\
\hline Variable & Descripción \\
\hline $\begin{array}{l}\text { Toma de decisiones } \\
\text { colectivas }\end{array}$ & $\begin{array}{l}\text { Consenso grupal para el bien común del grupo. } \\
\text { Una voz, un voto. }\end{array}$ \\
\hline $\begin{array}{l}\text { Movilidad por el } \\
\text { trabajo productivo }\end{array}$ & $\begin{array}{l}\text { Libertad para salir a trabajar. } \\
\text { Libertad para trasladarse fuera de la comunidad y } \\
\text { vender productos. } \\
\text { Libertad para viajar y promover/vender los productos. }\end{array}$ \\
\hline
\end{tabular}


(continuación)

\begin{tabular}{|l|l|}
\hline \multicolumn{2}{|l|}{ Dimensión sociocultural } \\
\hline $\begin{array}{l}\text { Equidad en la } \\
\text { comunidad }\end{array}$ & $\begin{array}{l}\text { Valoración del trabajo de las mujeres en la comunidad. } \\
\text { Conocimiento de los derechos de las mujeres. } \\
\text { Igualdad de oportunidades de trabajo para hombres } \\
\text { y mujeres. } \\
\text { Equidad de género entre niños y niñas. }\end{array}$ \\
\hline $\begin{array}{l}\text { Redes a partir } \\
\text { del grupo }\end{array}$ & $\begin{array}{l}\text { Agrupación libre de las mujeres del grupo. } \\
\text { Construcción de redes y apoyo entre las mujeres del } \\
\text { grupo. } \\
\text { Construcción de relaciones con compradores y } \\
\text { proveedores. }\end{array}$ \\
\hline
\end{tabular}

Fuente: elaboración propia a partir de las dimensiones propuestas por diversos autores: Alkire, et al. (2012), Foley, (1997), Gigler (2011), Haugh y Talwar (2014), Lemaître y Helmsing (2012), Malhotra, Schuler, Boender (2002), Zapata-Martelo y Mercado-González (1996).

\section{6. Índices de empoderamiento en la organización productiva}

Se obtienen los índices de empoderamiento de cada una de las mujeres participantes en los grupos productivos en las cuatro dimensiones: económica, personal, familiar y sociocultural; también se calculan los índices de empoderamiento por dimensión por grupo.

Se retoma la medición del Índice de Desarrollo Humano (IDH) del Programa de las Naciones Unidas para el Desarrollo, PNUD (2014) donde se señala que los índices son indicadores que van de 0 a 1; es decir, cuanto mayor empoderamiento se presente en las mujeres, el resultado será más cercano a la unidad; por el contrario, cuando el empoderamiento sea más bajo, el índice marcará un valor cercano a cero; para el análisis e interpretación de los índices, se consideran cinco niveles como se detalla en la siguiente tabla (ver tabla 6).

Tabla 6. Organización de resultados por niveles de empoderamiento

\begin{tabular}{|l|l|}
\hline \multicolumn{2}{|c|}{ Niveles de los índices de empoderamiento } \\
\hline Muy bajo & 0 a .200 \\
\hline Bajo & .201 a .400 \\
\hline Medio & .401 a .600 \\
\hline Alto & .601 a .800 \\
\hline Muy alto & .801 a 1 \\
\hline
\end{tabular}

Fuente: elaboración propia. 
El índice de empoderamiento se obtiene por la dimensión de cada uno de los grupos y, a partir de estos indicadores, se obtiene el índice de empoderamiento total por grupo. Se encontró que todos los grupos están empoderados: siete presentan un nivel de empoderamiento muy alto, cinco grupos tienen un nivel de empoderamiento alto y solo un grupo presenta un nivel medio. En la gráfica 1 se muestra el nivel de empoderamiento para cada grupo. En color gris oscuro se señalan los que tienen un índice entre .801 - 1 (muy alto); en gris medio, los que presentan un índice entre .601 - 800 (alto), y en blanco aquellos cuyo índice de empoderamiento está entre .402 - .600 (medio). (Ver gráfica 1)

Gráfica 1. Comparación gráfica de los niveles de los índices de empoderamiento en las cuatro dimensiones de estudio

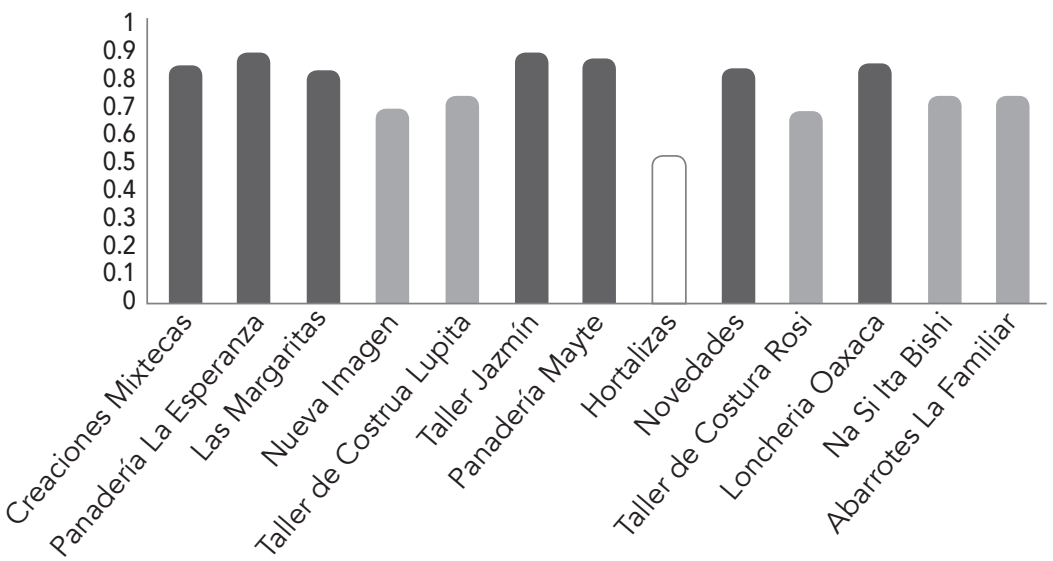

Fuente: elaboración propia a partir del levantamiento de datos.

En la tabla 7 se presenta el índice de empoderamiento de cada uno de los grupos en las cuatro dimensiones (ver tabla 7).

Tabla 7. Índice de empoderamiento por dimensión de cada grupo de trabajo

\begin{tabular}{|l|c|c|c|c|}
\hline Nombre & $\begin{array}{c}\text { Dimensión } \\
\text { económica }\end{array}$ & $\begin{array}{l}\text { Dimensión } \\
\text { personal }\end{array}$ & $\begin{array}{l}\text { Dimensión } \\
\text { familiar }\end{array}$ & $\begin{array}{l}\text { Dimensión } \\
\text { sociocultural }\end{array}$ \\
\hline Creaciones Mixtecas & .87 & .90 & .83 & .77 \\
\hline P. La Esperanza & .82 & .82 & .94 & .98 \\
\hline Taller de Uniformes & .78 & .87 & .84 & .80 \\
\hline Nueva Imagen & .86 & .80 & .61 & .50 \\
\hline
\end{tabular}


(continuación)

\begin{tabular}{|l|c|c|c|c|}
\hline Nombre & $\begin{array}{l}\text { Dimensión } \\
\text { económica }\end{array}$ & $\begin{array}{l}\text { Dimensión } \\
\text { personal }\end{array}$ & $\begin{array}{l}\text { Dimensión } \\
\text { familiar }\end{array}$ & $\begin{array}{l}\text { Dimensión } \\
\text { sociocultural }\end{array}$ \\
\hline $\begin{array}{l}\text { Taller de Costura } \\
\text { Lupita }\end{array}$ & .71 & .73 & .76 & .76 \\
\hline Taller Jazmín & .82 & .94 & .92 & .89 \\
\hline Panadería Mayte & .88 & .87 & .86 & .89 \\
\hline S. y C. de Hortalizas & .53 & .43 & .54 & .59 \\
\hline Novedades & .82 & 1 & .94 & .58 \\
\hline Taller de Costura Rosi & .68 & .70 & .72 & .63 \\
\hline Lonchería Oaxaca & .85 & .77 & .93 & .84 \\
\hline Na Si Ita Bishi & .61 & .71 & .80 & .82 \\
\hline Abarrotes La Familiar & .73 & .66 & .89 & .69 \\
\hline
\end{tabular}

Fuente: elaboración propia a partir del levantamiento de datos.

\section{Análisis de los resultados}

La medición del empoderamiento a través de índices permite corroborar que las mujeres indígenas integrantes de organizaciones productivas entran en un proceso de empoderamiento a partir de la labor realizada en los grupos de trabajo. Al analizar los reactivos de cada una de las dimensiones, se valoran aspectos de manera detallada.

En cuanto a la dimensión económica, consideran que tener un ingreso propio es importante pues les confiere independencia; empiezan a tomar decisiones dentro del hogar en cuanto a la repartición del gasto, dado que son ellas quienes administran el dinero que reciben al participar en el grupo productivo; ello les da autonomía; sienten reconocimiento de la familia y aprecian poder hacer compras para sus hijas e hijos.

En cuanto a la dimensión personal, expresan tener mayor confianza en sí mismas y seguridad al tomar sus propias decisiones. El trabajo productivo les confiere certeza en los gastos, aun si estos se destinan mayoritariamente a los hijos. Afirman tener la libertad de salir del hogar para trabajar, aunque la mayoría debe pedir «permiso» a su pareja.

En la dimensión familiar, en todos los casos manifiestan que, al principio, la pareja no está de acuerdo con su participación en el grupo de trabajo; sin embargo, las mujeres persisten en su interés de participar y encuentran la manera de lograr su objetivo. Aplicar el instrumento de manera personalizada permite indagar acerca del motivo de deserción de las otras mujeres de los grupos, que en todos los casos se relaciona con el trabajo de cuidados, 
es decir, el cuidado y administración del hogar, alimentación de la familia, crianza de las(os) hijas(os) o personas mayores. Es de notar que todas las mujeres están de acuerdo con la importancia de la educación para sus hijas(os) sin distinción por sexo. Tanto hombres como mujeres deben prepararse y asistir a la escuela.

En cuanto a la dimensión sociocultural, las mujeres aseguran que pertenecer al grupo les da seguridad, y les permite compartir sus experiencias y escuchar las de otras mujeres; sienten apoyo. Expresan que las decisiones en el grupo se toman de manera conjunta, la voz de todas se toma en cuenta y es igual de importante. Consideran que sí hay reconocimiento de su trabajo en la comunidad.

Se genera valor social por su participación en el proyecto productivo en tanto que sí se manifiesta un proceso de empoderamiento en todos los grupos de trabajo. Se inicia una transformación personal que impacta en la familia, cambia la apreciación de las dinámicas socioculturales; sin embargo, el ingreso económico es valorado como un apoyo; solo en los grupos Creaciones Mixtecas y Taller de costura Jazmín manifiestan que el ingreso es suficiente y constante.

Los resultados coinciden con la investigación de Malhotra, Schuler y Boender (2002), quienes resaltan la heterogeneidad de los procesos de empoderamiento, mismos que, explican, pueden presentarse en una dimensión y no manifestarse en las otras o presentarse en diferente nivel; asimismo, Rowlands (1997) añade que puede tener diferentes niveles de intensidad, no solo en dimensiones o niveles distintos. Aguilar-Pinto, et al. (2017) señalan que el empoderamiento se concentra en la dimensión personal, más que en la colectiva y de relaciones cercanas.

\section{Conclusión}

Se observa que las mujeres indígenas de los grupos de trabajo de mujeres indígenas apoyadas por el PROIN, en Ensenada, han tenido un proceso de empoderamiento de 2014 a 2017, en diferente nivel, en cada una de las dimensiones de estudio; sin embargo, es evidente una alta deserción del proyecto productivo. Las mujeres que permanecen en estos potencian sus niveles de empoderamiento. Desafortunadamente, el nivel de deserción es elevado sobre todo debido a las responsabilidades que las mujeres asumen como propias, siendo que la sociedad les impone, en el hogar, el trabajo de cuidados de un sistema patriarcal.

Desempeñar el trabajo productivo supone para ellas tener que negociar con sus familiares y parejas, quienes consideran que el lugar de las mujeres es el hogar y su responsabilidad, la crianza de las(os) hijas(os), la alimentación 
y el cuidado de la familia. Esta situación ocasiona conflicto y presión, por lo que la mayoría abandona el grupo de trabajo.

Aquellas que persisten deben realizar ambos tipos de trabajo, lo que inevitablemente las conduce a una doble jornada y a cansancio y desgaste como resultado de una baja participación de los hombres en el trabajo de cuidados. Quienes continúan, lo hacen principalmente debido a la generación de un proceso de empoderamiento que transforma sus percepciones personales y colectivas, identifican nuevos beneficios: construyen redes, incrementan la seguridad con la que toman decisiones, reafirman los beneficios del trabajo, y adquieren o incrementan su nivel de libertad de movimiento en el espacio público.

Debido a ello, es necesario considerar los conflictos basados en las costumbres socioculturales de las mujeres y familias beneficiarias para el diseño de los programas de política pública que pretenden conducir a las mujeres indígenas a un proceso de empoderamiento a través de proyectos productivos. Las costumbres socioculturales se basan en un sistema patriarcal, en el cual la posición y condición de las mujeres está supeditada a los hombres. Su participación en el grupo y el ingreso económico puede conducir a problemas familiares en donde otras mujeres u hombres del grupo doméstico extensivo podrían inhibir su desarrollo.

Destaca la necesidad de un programa de sensibilización con perspectiva de género que incluya a unas y otros. Es importante que este se realice de manera paralela a la participación en los grupos productivos, desde la solicitud del recurso, hasta la consolidación de los proyectos productivos. Dada la situación de inequidad extendida en la mayoría de las mujeres, esta acción es obligada para, efectivamente, coadyuvar en los procesos de empoderamiento de las mujeres indígenas. El acompañamiento cercano y constante para guiar a las mujeres es un factor que posibilita el avance hacia una transformación social equitativa, el cual, brindado por personal sensible al enfoque de género, resulta determinante dada la situación patriarcal existente.

La medición del empoderamiento a través de índices multidimensionales, tal como se ha evidenciado en este trabajo, permite generalizar resultados y evaluar el avance de programas productivos cuyo objetivo es el empoderamiento de las mujeres. Por ello, se recomienda la aplicación de instrumentos, como el planteado, para evaluar los programas de políticas públicas y contribuir a su mejora, lo cual ha de ir acompañado de herramientas cualitativas que permitan ahondar en las áreas de oportunidad de la aplicación de políticas publicas y de empoderamiento de las mujeres indígenas, a través de su participación en grupos productivos. Esto es así debido a que los procesos de empoderamiento deben comprender y considerar no solo el aspecto económico, sino también cuestiones menos tangibles, las dinámicas de su cotidianeidad, su contexto, costumbres y tradiciones. 


\section{Referencias bibliográficas}

Aguilar-Pinto, C., Tuñón-Pablos, E., Zapata-Martelo, E. y Evangelista-García, A. (2017). «Women, Empowerment and microcredit: Banmujer's social microenterprise program in Chiapas». Agricultura, sociedad y desarrollo, vol. 14 (3): 479-501.

Aguinaga, M., Lang, M., Mokrani, D. y Santillana, A. (2011). «Pensar desde el feminismo: Críticas y alternativas de desarrollo. Grupo permanente de trabajo sobre alternativas al desarrollo». En Lang, M. y Mokrani, D. (comps.). Más allá del desarrollo. Grupo Permanente de Trabajo sobre Alternativas al Desarrollo. Quito: Fundación Rosa Luxemburg/Abya Yala. Recuperado el 6 de octubre de 2019 de: https://www.researchgate. net/publication/303543093

Alberti-Manzanares, P., Zavala-Hernández, M., Salcido-Ramos, B. y RealLuna, N. (2014). «Género, economía del cuidado y pago del trabajo doméstico rural en Jilotepec, Estado de México». Agricultura, sociedad y desarrollo, vol. 11 (3): 379-400. Recuperado el 6 de octubre de 2019 de: http://www.scielo.org.mx/scielo.php?script=sci_arttext\&pid $=\mathrm{S} 1870-54722014000300007$

Alkire, S., Meinzen-Dick, R., Peterman, A., Quisumbing, A., Seympur, G. y Vaz, A. (2012). «The Women's Empowerment in Agriculture index». EE.UU.: USAID, International Food Policy Research Institute IFPRI y The Oxford Poverty and Human Development Initiative, OPHI. Recuperado el 9 de octubre de 2019 de: http://www.ifpri.org/publication/ womens-empowerment-agriculture-index

Banerjee, S. y Hackson, L. (2017). «Microfinance and the business of poverty reduction: Critical perspectives from rural Bangladesh». Human Relations, vol. 70 (1): 63-91.10. 1177/0018726716640865

Consejo Nacional de Evaluación de la Política de Desarrollo Social, CONEVAL (2015) Medición de la pobreza en México y de las entidades federativas 2014. México: CONEVAL. Recuperado el 6 de octubre de 2019 de: http://www.coneval.org.mx/Medicion/Documents/Pobreza\%20 2014_CONEVAL_web.pdf

Díaz-Carrión, I. (2012). Género y turismo alternativo: aproximaciones al empoderamiento (tesis doctoral). Madrid: Universidad Complutense de Madrid. Recuperado el 6 de octubre de 2019 de: http://eprints.ucm. es/14733/

Díaz-Cervantes, R. (2015). «Estudios de género en organizaciones y emprendimientos de mujeres rurales en México». En Zapata-Martelo, E. y AyalaCarrillo, R. (coord.). Contribuciones de los estudios de género a desarrollo rural. México: Colegio de Postgraduados: 242-270. 
Foley, J. (1997). «The Dimensions of Empowerment». 29 Annual International Conference of the Community Development Society, Atenas, Grecia Recuperado el 6 de octubre de 2019 de: https://files.eric.ed.gov/fulltext/ ED413514.pdf

García-Canclini, N. (2012) Culturas híbridas. México: Grijalbo.

García-Horta, J. y Zapata, E. (2012). «El papel de las micro-finanzas en la pobreza y desigualdad de las mujeres». Ra Ximhai. Revista de Sociedad, Cultura y Desarrollo Sustentable, vol. 8 (1): 101-111. Recuperado el 6 de octubre de 2019 de: http://www.redalyc.org/pdf/461/46123324007.pdf

Gigler, B. (2011). «Informational Capabilities - The Missing Link for the Impact of ICT on Development» (en línea). Open Development technology Alliance. 10.2139/ssrn.2191594

Gil, L: (2017). «Pensamiento feminista y política transformadora, una aproximación». Revista Laguna (40): 107-119. Recuperado el 6 de octubre de 2019 de: http://riull.ull.es/xmlui/handle/915/7071

Gobierno de la República, Secretaría de Desarrollo Social (2013). Programa Sectorial de Desarrollo Social, PSDS, 2013-2018. México: Gobierno de la República. Recuperado el 6 de octubre de 2019 de: https://bit.ly/1H7zWH4

Gobierno de la República y Comisión Nacional para el Desarrollo de los Pueblos Indígenas (2014). Programa Especial de los Pueblos Indígenas, 2014-2018. México: CDI, 2014. Recuperado el 16 de octubre de 2019 de: https:/www.gob.mx/cms/uploads/attachment/file/32305/cdi-programaespecial-pueblos-indigenas-2014-2018.pdf

Gobierno de la República, Comisión Nacional para el Desarrollo de los Pueblos Indígenas (2017). Acuerdo por el que se modifican las Reglas de Operación del Programa para el Mejoramiento de la Producción y Productividad Indígena a cargo de la Coordinación General de Fomento a la Producción y Productividad Indígena de la Comisión Nacional para el Desarrollo de los Pueblos Indígenas para el ejercicio fiscal 2018. Diario Oficial de la Federación, 28 de diciembre de 2017. Recuperado el 6 de octubre de 2019 de: https://bit.ly/31WuTe2

Haugh, H. y Talwar, A. (2016). «Linking Social Entrepreneurship and Social Change: The Mediating Role of Empowerment». Journal of Business Ethics, vol. 133 (4): 643-658. 10.1007/s10551-014-2449-4

Kabeer, N (1999). «Resource, Agency, Achievements: Reflections on the Measurement of Women's Empowerment». Development and Change, vol. 30: 435-464. Recuperado el 6 de octubre de 2019 de: https://bit.ly/2buO3xL

Kabeer, N (2001). «Conflicts over credit: Re-Evaluating the empowerment potential of loans to women in rural Bangladesh». World Development, 29 (1): 63-84. Recuperado el 6 de octubre de 2019 de: https://bit.ly/3225gaH Lagarde, M. (2005). Los cautiverios de las mujeres: madresposas , monjas, putas, presas y locas. México: Universidad Nacional Autónoma de México. 
Lemaitre, A. y Helmsing, A. (2012). «Solidarity economy in Brazil: Movement, discourse and practice analysis through a Polanyian understanding of the economy». Journal of International Development (24): 745-762. $10.1002 /$ jid. 2865

Longwe, S. y Clarke, R. (1994). Women in Development, Culture and Youth. Workshop Preparatory Readings. Lusaka: Longwe Clarke Associates

Lukes, S. (2005). Power: A radical view. Basingstoke: Palgrave Macmillan.

Malhotra, A., Schuler, S. y Boender, C. (2002). «Measuring Women's empowerment as a variable in international development» (documento de trabajo). World Bank Workshop on Poverty and Gender: New Perspectives, s.p.i. Recuperado el 6 de octubre de: https://siteresources.worldbank.org/ INTGENDER/Resources/MalhotraSchulerBoender.pdf

Martínez-Corona, B. (2000). Género, empoderamiento y sustentabilidad, una experiencia de microempresa artesanal de mujeres indigenas. México: Grupo Interdisciplinario sobre Mujer, Trabajo y Pobreza, A.C.

Mathur, P. y Agarwal, P. (2016). «Self-help groups: a seed for intrinsic empowerment of Indian rural women», Equality, Diversity and Inclusion: An International Journal, vol. 36 (2): 182-196. 10.1108/EDI-05-2016-0039

Mayoux, L. (2000). Microfinance and the empowerment of women: A review of the key issues. Ginebra: ILO, Social Finance Unit. Recuperado el 6 de octubre de: https://www.ilo.org/public/libdoc/ilo/2000/100B09_285_ engl.pdf

Meza Ojeda, A., Tuñón Pablos, E., Ramos Muñoz, D., Kauffer, M. y Kauffer, E. (2002). «Progresa y el empoderamiento de las mujeres: estudio de caso en Vista Hermosa, Chiapas». Papeles de Población, Universidad Autónoma del Estado de México, vol. 8 (31): 67-93.

Organización de las Naciones Unidas, ONU (2007) Declaración sobre los Derechos de los Pueblos Indígenas. Recuperado el 16 de octubre de 2019: https:// www.un.org/development/desa/indigenous-peoples-es/declaracion-sobrelos-derechos-de-los-pueblos-indigenas.html

Organización de las Naciones Unidas, ONU (2015). Objetivos de Desarrollo del Milenio, informe de 2015. Recuperado el 6 de octubre de 2019 de: http://mdgs.un.org/unsd/mdg/Resources/Static/Products/Progress2015/ Spanish2015.pdf

Organización de las Naciones Unidas, ONU (2015), Objetivos de Desarrollo Sostenible (ODS) (en línea). Recuperado el 6 de octubre de 2019 de: https://www.un.org/sustainabledevelopment/es/2015/09/la-asambleageneral-adopta-la-agenda-2030-para-el-desarrollo-sostenible/

Pont, E. (2010). «Yo no me siento pobre». La ventana. Revista de estudios de género, vol. 4 (31): 36-63. Recuperado el 6 de octubre de 2019 de: https://bit.ly/2jZoQUT 
Programa de las Naciones Unidas para el Desarrollo, PNUD (2014). Indicadores de desarrollo humano y género en México: nueva metodología (s.p.i.). Recuperado el 16 de octubre de 2019 de: https:/www.undp.org/ content/dam/mexico/docs/Publicaciones/PublicacionesReduccionPobreza/ InformesDesarrolloHumano/DHyG\%20baja\%20res.pdf

Rahman, A. (2013). «Women's Empowerment: Concept and Beyond». Global Journal of Human Social Science, Sociology and Culture, 13 (6): 8-13. Recuperado el 6 de octubre de 2019 de: https://globaljournals.org/ GJHSS_Volume13/2-Womens-Empowerment-Concept.pdf

Ramírez-Salgado, R. (2017). «Educación para los medios y feminismo: una articulación que posibilita el empoderamiento de las mujeres». Communication papers, media literacy and Gender studies, vol. 5 (10): 59-70. Recuperado el 16 de octubre de 2019 de: https://www.academia.edu/36769352/ Contacto_Art\%C3\%ADculo_Educaci\%C3\%B3n_para_los_medios_y_ feminismo_una_articulaci $\% \mathrm{C} 3 \% \mathrm{~B} 3 \mathrm{n}$ que posibilita_el_empoderamiento_ de las mujeres

Rivas-Ángeles, K., Alberti-Manzanares, P., Osnaya, M. y León-Merino, A. (2015). «Mujeres rurales: del proyecto productivo a la microempresa en Champotón, Campeche». Revista Mexicana de Ciencias Agrícolas, 6(6): 1359-1371. Recuperado el 9 de octubre de 2019 de: https://bit. ly/2CqyUv8

Rodríguez-Enríquez, C. (2015). «Economía feminista y economía del cuidado, aportes conceptuales para el estudio de la desigualdad». Revista Nueva Sociedad, 256: 30-44. Recuperado el 16 de octubre de 2019 de: https://nuso.org/media/articles/downloads/4102_1.pdf

Rowlands, J. (1997). «Empoderamiento y mujeres rurales en Honduras: un modelo para el desarrollo». En León, M. (comp.). Poder y empoderamiento de las mujeres. Bogotá: Tercer Mundo: 213-245.

Serrano-Barquín, R. del C., Serrano Barquín, R. A,. Palmas Castrejón, D. y Cruz Jiménez, G., (2013). «La participación de la mujer en el desarrollo local de comunidades indígenas a partir de proyectos turísticos». En Serrano-Barquín, C. y Rojas-García, A. (coord.). Mas allá del imaginario eurocéntrico empoderamiento de la mujer indígena. México: Publicaciones Administrativas Contables Jurídicas.

Sistema de Clasificación Industrial de Amércia del Norte, SCIAN (2018). Recuperado el 16 de octubre de 2019 de: https://www.inegi.org.mx/app/scian/

Stromquist, N. (1997). «La búsqueda del empoderamiento: en qué puede contribuir el campo de la educación». En León, M. (comp.). Poder y empoderamiento de las mujeres. Colombia: Tercer Mundo: 75-95.

Tuñón-Pablos, E. (2010). «Evaluación de los programas de crédito a proyectos productivos de mujeres en Tabasco, Campeche y Quintana Roo». La 
Ventana, Revista de Estudios de Género, vol. 32 (3): 81-116. Recuperado el 6 de octubre de: http://www.redalyc.org/pdf/884/88420963005.pdf

Virgilí, P. (2014). «Reflexiones teórico-metodológicas sobre sensibilización y capacitación en género. Apuntes de una propuesta para su implementación en contextos grupales». La ventana. Revista de Estudios de Género, vol. 5 (40): 7-58.

Young, K. (1997). «El potencial transformador en las necesidades prácticas: empoderamiento colectivo y el proceso de planificación». En León, M. (comp.). Poder y empoderamiento de las mujeres. Colombia: Tercer Mundo: 99-118. Yunus, M. (2007). Creating a world without poverty, Social Business and the Future of Capitalism. Nueva York: Public Affairs.

Zapata-Martelo, E. y Mercado-González, M. (1996). «Del proyecto productivo a la empresa social de mujeres». En Canabal, B. y Espinosa, G. (coord.). La mujer en el medio rural. México: Cuadernos Agrarios: 104-128.

Zarza-Delgado, M. P., Serrano-Barquín, H. (2013). «Empoderamiento de la mujer mazahua y otomí a través del diseño textil». En Serrano-Barquín, C. y Rojas-García, A. (coords.). Más allá del imaginario eurocéntrico. Empoderamiento de la mujer indígena. México: Publicaciones Administrativas Contables Jurídicas.

Zapata, E., Vázquez, V., Alberti, P., Pérez, E., López, J., Flores, A., Hidalgo, N. y Garza, E. (2008). Microfinanciamiento y empoderamiento de mujeres rurales, las cajas de ahorro y crédito en México. México: Plaza y Valdés.

\section{Obras consultadas}

Benería, L. (2016). ¿Hacia un super-neoliberalismo?: EL TTIP (archivo de video). Conferencia en las jornadas de «La superglobalización, una perspectiva desde las migraciones internacionales», 25 de mayo de 2015, Facultad de Sociología de la Universidade da Coruña. Recuperado el 10 de octubre de 2019 de: https://www.youtube.com/watch?v=RZ70v4KOuyU

Meza, A., Tunón, E., Ramos, D. y Michel, E. (2002). «Progresa y el empoderamiento de las mujeres: estudio de caso en Vista Hermosa, Chiapas». Papeles de Población, 8 (31): 67-93. Recuperado el 10 de octubre de 2019 de: http://www.redalyc.org/pdf/112/11203103.pdf

Stavenhagen, R. (2007). Los pueblos indígenas y sus derechos. México: UNESCO México. 


\section{Los autores}

Diana G. Robinson-Trápaga es candidata a doctora en Ciencias Administrativas por la Universidad Autónoma de Baja California, maestra en Mercadotecnia por el CETYS, Universidad Ensenada, y licenciada en Diseño Gráfico por la Universidad Iberoamericana Santa Fe. Imparte clases a nivel de licenciatura en la Escuela de Administración y Negocios y en Ingeniería en Diseño Gráfico Digital en CETYS Universidad y a nivel posgrado en la Universidad Iberoamericana. Cuenta con publicaciones nacionales e internacionales, sus líneas de investigación son el emprendimiento social y el empoderamiento de la mujer. Dirige su propio estudio de diseño y mercadotecnia desde 1998 a la fecha.

diana.robinson@uabc.edu.mx

Isis Arlene Díaz Carrión es doctora en Geografía Humana por la Universidad Complutense de Madrid, cuenta con un máster en Gestión Pública del Turismo por la Universidad Internacional de Andalucía, una maestría en Geografía (revalidación SEP) y es licenciada en Administración de Empresas por la Universidad Veracruzana. Actualmente se desempeña como profesora-investigadora de la Facultad de Turismo y Mercadotecnia de la Universidad Autónoma de Baja California, donde imparte clases de licenciatura y posgrado. En su labor de formación de recursos humanos ha dirigido diversas tesis de posgrado y supervisado ayudantías de investigación a nivel licenciatura. Ha sido responsable y colaboradora en varios proyectos de investigación. Sus líneas de investigación son turismo y sostenibilidad y turismo y género. Cuenta con diversas publicaciones nacionales e internacionales en esos temas. Forma parte del consejo editorial de diversas revistas nacionales e internacionales.

diaz.isis@uabc.edu.mx

Sergio Cruz-Hernández estudió Ciencias de la Comunicación en 1998 y la maestría en Ciencias Sociales en 2008, en la Universidad Autónoma de Baja California. Desde 1998 se involucró con organizaciones de la sociedad civil en el area de comunicación, en temas de grupos indígenas y conservación para el desarrollo sostenible, razón por la cual ha realizado proyectos sociales con pescadores y grupos rurales. Tiene más de veinte años de experiencia como reportero, creativo en agencias de publicidad, guionista, diseñador gráfico y de páginas web, ilustrador, productor de 
radio, realizador de video documental y músico. Es doctor en Ciencias y Humanidades para el Desarrollo Interdisciplinario por el Centro de Investigaciones Interdisciplinarias en Ciencias y Humanidades de la UNAM y la Universidad de Coahuila, en 2015. Desde 2005 es profesor en la Facultad de Ciencias Administrativas y Sociales y actualmente se desempeña como director para el período 2017-2021. Como profesor investigador pertenece al Sistema Nacional de Investigadores Nivel 1 y sus líneas de trabajo incluyen la comunicación para el desarrollo, las identidades sociales y la cibercultura.

checoch@uabc.edu.mx 